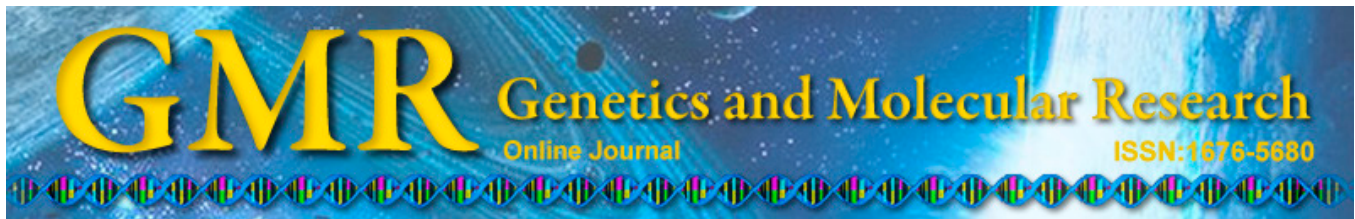

\title{
Clinical observation of umbilical cord mesenchymal stem cell treatment of severe systolic heart failure
}

\author{
X.F. Zhao, Y. Xu, Z.Y. Zhu, C.Y. Gao and Y.N. Shi \\ Department of Internal Medicine, Henan Provincial People's Hospital, \\ Zhengzhou, China \\ Corresponding author: Y. Xu \\ E-mail: yxxfen@126.com
}

Genet. Mol. Res. 14 (2): 3010-3017 (2015)

Received March 17, 2014

Accepted July 8, 2014

Published April 10, 2015

DOI http://dx.doi.org/10.4238/2015.April.10.11

\begin{abstract}
The aim of this study was to evaluate the effectiveness of umbilical cord mesenchymal stem cells (MSCs) in the treatment of chronic systolic heart failure. Fifty-nine hospitalized patients with heart failure were randomly divided into a treatment group (30 patients) and a control group (29 patients). The treatment group received treatment with medication as well as intracoronary transplantation of umbilical cord MSCs, and the control group, only medication. The cardiac structure, function change, and rehospitalization and mortality rates of the 2 groups were observed before and 1 and 6 months after treatment. One month after the transplantation of umbilical cord MSCs, the incidence of fatigue, chest tightness, and dyspnea was high in the treatment group. The 6-min walking distance of the treatment group was found to be significantly higher than that of the control group $(\mathrm{P}<0.05)$; in addition, the NT-proBNP level, left ventricular ejection fraction, and mortality rate of the treatment group were statistically lower than those of the control group $(\mathrm{P}<0.05)$. Readmission rates showed a downward trend, but the difference was not statistically significant $(\mathrm{P}>0.05)$. Using umbilical cord MSCs in the treatment of congestive heart failure
\end{abstract}


can help improve cardiac remodeling and cardiac function and reduce the mortality rate.

Key words: Umbilical cord; Mesenchymal stem cells (MSCs); Systolic heart failure; MSC effectiveness

\section{INTRODUCTION}

Heart failure is a chronic disease and the end stage of various heart diseases. It is a progressive syndrome that results in a poor quality of life for the patient and places an economic burden on the health care system. Once heart failure occurs, it continues to develop even in the absence of new pathogenic factors and causes serious harm to the patient (Ambrosy et al., 2014). The hospitalization and mortality rates related to heart failure are high (Sbruzzi et al., 2010). Over the last decade, the annual number of heart failure hospitalizations has almost doubled, with approximately $50 \%$ of the patients rehospitalized within 6 months of discharge (Giamouzis et al., 2011). In China, the incidence of heart failure is found to increase every year. Heart failure is also considered as the final battle against heart disease.

Heart failure is currently very difficult to treat. Treatment with drugs can, to a certain extent, slow or stop the progression of heart failure and save dying cardiomyocytes, but no beneficial effect of treatment is seen on myocardial necrosis. Another effective method for treating heart failure is cardiac transplantation (Feldman et al., 2013), but because of the low availability of donor hearts, high cost of surgery, and a high likelihood of immune rejection, it is difficult to perform heart transplantations (Chih et al., 2013).

The main pathological characteristic of heart failure is the loss of function of the myocardium, so stem cell regeneration in myocardial tissue may be a new method of improving the prognosis of patients. Stem cell transplantation is a new method for the biological treatment of heart failure (Perin et al., 2012a). Stem cells can promote proliferation, differentiate in the myocardium, replace the necrotic myocardial cells that have lost function, strengthen and improve heart function, and promote ventricular remodeling (Perin et al., 2012b), thus preventing the progression of heart failure.

Umbilical cord mesenchymal stem cells (MSCs) are widely available, promote proliferation and differentiation, have low immunogenicity, and can be collected in a noninvasive manner; therapy involving MSCs is attracting increasing attention. Animal experiments involving treatment with umbilical cord MSCs have confirmed that the cells can be converted into myocardial cells (Chugh et al., 2012) and endothelial progenitor cells (Hou and Li, 2007). However, only a few cases of the clinical application in the treatment of decompensated congestive heart failure have been reported. The use of bone marrow MSCs in the treatment of congestive heart failure had obvious clinical effect at previous time of this study, improved heart function. This study examined the effects of umbilical cord MSC transplantation on cardiac function and clinical prognosis in patients with congestive heart failure.

\section{MATERIAL AND METHODS}

\section{Subjects}

All the 59 patients were hospitalized at the Cardiovascular Department of Inter- 
nal Medicine, Henan Province People's Hospital, between December 2010 and December 2011. The inclusion criteria were as follows: 1) age 18-80 years; 2) clinically diagnosed decompensated congestive heart failure, cardiac function III or IV (New York Heart Association grade) of dilated cardiomyopathy or ischemic cardiomyopathy; 3) left ventricular end-diastolic diameter (LVEDD): male $\geq 55 \mathrm{~mm}$, female $\geq 50 \mathrm{~mm}$; 4) left ventricular ejection fraction (LVEF) $\leq 35 \%$; and 5 ) $N$-terminal pro-brain natriuretic peptide (NT-proBNP) level $\geq 1500 \mathrm{pg} / \mathrm{mL}$ measured 2 weeks before inclusion. The exclusion criteria were as follows: valvular heart disease, hypertrophic cardiomyopathy, restrictive cardiomyopathy, congenital heart disease, acute viral myocarditis, pacemaker implantation, acute coronary syndrome, malignant tumor, and severe liver and kidney dysfunction; the diagnoses were in accordance with the guidelines of the 2007 chronic heart failure diagnostic criteria. Finally, 59 cases were randomly divided into the control group (29 cases) and treatment group (30 cases). The control group was treated only with drugs. The treatment group was treated with drug therapy as well as intracoronary injection of umbilical cord MSCs. This study was conducted in accordance with the Declaration of Helsinki. This study was approved by the Ethics Committee of Henan Provincial People's Hospital, and written informed consent was obtained from all participants.

\section{Preparation and transplantation of umbilical cord MSCs}

Healthy full-term fetal umbilical cord was used, with the parent's consent and approval of the Ethics Committee, for the record. In the center of Henan Province People's Hospital, umbilical cord MSCs were obtained by the tissue culture method (Weiss et al., 2006; Xu et al., 2009; Li et al., 2011) and marked as CD29 and CD105 by flow-cell immune phenotype. Hematopoietic stem cells with no expression or low expression were specifically marked as CD34, CD45, pyrogen monitoring, active degree $\geq 95 \%$, weight approximately $20 \mathrm{~mL}$. With the patient in the stable condition, two-thirds of the umbilical cord MSCs were injected into left coronary artery, and one-third, into the right coronary artery, through the coronary artery.

\section{Observation index}

At the 1- and 6-month follow-up, the patients' vital signs, electrocardiographic findings, cardiac ultrasonography heartbeat graph, NT-proBNP levels, 6-min walking distance, clinical symptoms (dyspnea, edema, exercise tolerance, bloating, and anorexia) were monitored, and the rehospitalization and mortality rates were noted at the 6-month follow-up.

\section{Statistical analysis}

The SPSS 17 statistical software was used for the following analysis: normal distribution of measurement data reported as means \pm standard deviation, non-normal distributions were transformed into normal using a logarithmic transformation, comparison of data between the groups was made by the independent sample $t$-test, and comparison of data before and after treatment was made by the paired $t$-test. Count data are reported as percentage, by the $\chi^{2}$ test. A P value $<0.05$ was set as statistically significant. 


\section{RESULTS}

\section{Baseline data}

Fifty-nine patients completed the follow-up, 43 men and 16 women with ages that ranged from 20 to 79 years (mean age, $55.02 \pm 14.18$ years). Nineteen patients had ischemic cardiomyopathy (32.2\%), and 40 patients, dilated cardiomyopathy (67.8\%). Comparison of general data before treatment between the treatment group and the control group is shown in Table 1.

Table 1. Comparison of general data before treatment between the treatment group and the control group.

\begin{tabular}{|c|c|c|c|c|}
\hline Group & Control & Treatment & $t$ value & $P$ value \\
\hline \multicolumn{5}{|l|}{ Gender } \\
\hline Male & 19 & 24 & & $>0.05$ \\
\hline Female & 10 & $6^{*}$ & & \\
\hline Age (years) & $53.21 \pm 11.46$ & $52.90 \pm 16.32$ & 2.696 & 0.106 \\
\hline \multicolumn{5}{|l|}{ Disease } \\
\hline Ischemic cardiomyopathy & 6 & 13 & & $>0.05$ \\
\hline Dilated cardiomyopathy & 23 & $17 * *$ & & \\
\hline $\operatorname{LVESD}(\mathrm{mm})$ & $59.14 \pm 7.44$ & $57.80 \pm 10.39$ & 2.292 & 0.136 \\
\hline $\operatorname{LVEDD}(\mathrm{mm})$ & $70.62 \pm 6.43$ & $69.53 \pm 9.28$ & 2.643 & 0.110 \\
\hline LVEF & $0.28 \pm 0.049$ & $0.30 \pm 0.045$ & 0.196 & 0.659 \\
\hline NT-proBNP (pg/mL) & $4701.76 \pm 513.53$ & $4376.27 \pm 510.71$ & 0.014 & 0.905 \\
\hline Six-minute walking distance $(\mathrm{m})$ & $369.97 \pm 82.05$ & $342.17 \pm 18.42$ & 4.248 & 0.078 \\
\hline
\end{tabular}

*Before treatment, age, LVESD, LVEDD, LVEF, NT-proBNP level, and 6-min walking distance showed no statistical difference $(\mathrm{P}>0.05)$ between the treatment group and control group. ${ }^{*}$ Gender differences assessed using the $\chi^{2}$ test; no statistical significance $(\mathrm{P}>0.05)$.

\section{Comparison of medicines used in the groups}

The patients with systolic heart failure were critically ill patients, with cardiac function III or IV (NYHA grade); the treatment group and control group patients were treated with $\beta$-blocker, angiotensin-converting enzyme inhibitors or angiotensin receptor blockers, diuretics, aldosterone receptor antagonists, and digoxin, provided their blood pressure was $\leq 90 \mathrm{mmHg}$, resting heart rate was $\leq 55 \mathrm{bpm}$, and they had no second-degree atrioventricular block and other contraindications (Table 2).

Table 2. Medicines administered to the treatment and control groups.

\begin{tabular}{lccr}
\hline Group & Control & Treatment & P value \\
\hline Digoxin (N) & 26 & 24 & $>0.05$ \\
Diuretics (N) & 26 & 25 & $>0.05$ \\
Aldosterone receptor antagonist (N) & 24 & 28 & $>0.05$ \\
ACEI/ARB (N) & 28 & 29 & $>0.05$ \\
Beta blockers (N) & 28 & 29 & $>0.05$ \\
\hline
\end{tabular}

Comparison between the 2 groups, using the $\chi^{2}$ test; no significant difference was observed $(\mathrm{P}>0.05)$; ACEI $=$ angiotensin-converting enzyme inhibitors; $\mathrm{ARB}=$ angiotensin receptor antagonist; $\mathrm{N}=$ number of cases.

\section{Changes in cardiac structure}

LVEDDs were statistically different between groups 6 months after transplantation ( $\mathrm{P}$ $<0.05)$, with no differences 1 month after transplantation ( $\mathrm{P}>0.05$; Table 3$)$. 
Table 3. LVEDD $(\mathrm{mm})$ changes before and 1 and 6 months after operation in the treatment group and the control groups.

\begin{tabular}{|c|c|c|c|c|}
\hline Group & Control $(\mathrm{N}=29)$ & Treatment $(\mathrm{N}=30)$ & $t$ value & P value \\
\hline Before surgery & $70.62 \pm 6.43$ & $69.53 \pm 9.28$ & 2.643 & 0.110 \\
\hline One month & $65.29 \pm 5.84$ & $61.93 \pm 8.04^{*}$ & 3.199 & 0.079 \\
\hline Six months & $63.13 \pm 6.40$ & $56.39 \pm 5.17^{* *}$ & 17.324 & $<0.05$ \\
\hline
\end{tabular}

LVEDDs for the control and treatment groups showed no significant difference before and 1 month after the surgery $(P>0.05)$. Six months after the surgery, the difference was statistically significant $(P<0.05)$. *LVEDDs for the treatment group before and 1 month after surgery were statistically significant $(\mathrm{P}<0.05) ;{ }^{* *}$ values before and 6 months after surgery $(\mathrm{P}<0.01)$.

\section{Changes in LVEF}

LVEF was statistically different between groups 1 and 6 months after transplantation $(\mathrm{P}<0.05)$, with no differences before transplantation $(\mathrm{P}>0.05$; Table 4$)$.

Table 4. LVEF changes in the treatment group and the control group before and 1 and 6 months after surgery.

\begin{tabular}{lcccc}
\hline Group & Control & Treatment & $t$ value & P value \\
\hline Preoperative & $0.28 \pm 0.051$ & $0.30 \pm 0.045$ & 0.196 & 0.659 \\
One month & $0.35 \pm 0.044$ & $0.46 \pm 0.058$ & 59.585 & $<0.01$ \\
Six months & $0.39 \pm 0.035$ & $0.49 \pm 0.051$ & 70.075 & $<0.01$ \\
\hline
\end{tabular}

Comparison of LVEF between the treatment group and the control group before surgery showed no statistical significance $(\mathrm{P}>0.05)$; values 1 month and 6 months after surgery were statistically difference $(\mathrm{P}<0.01)$.

\section{Changes in NT-proBNP level}

The NT-proBNP levels were statistically different between the groups 1 month after transplantation $(\mathrm{P}<0.05)$ and 6 months after transplantation $(\mathrm{P}<0.01)$, and no differences were observed before transplantation $(\mathrm{P}>0.05$, Table 5).

Table 5. Changes in NT-proBNP levels $(\mathrm{pg} / \mathrm{mL})$ between the treatment group and the control group before and
1 and 6 months after surgery.
\begin{tabular}{lccrr}
\hline Group & Control & Treatment & $t$ value & P value \\
\hline Preoperative & $4701.76 \pm 513.50$ & $4376.51 \pm 510.71$ & 0.014 & 0.905 \\
One month & $4142.32 \pm 488.10$ & $2517.04 \pm 413.79$ & 6.892 & 0.011 \\
Six months & $2835.09 \pm 412.03$ & $1648.96 \pm 304.54$ & 21.469 & $<0.01$ \\
\hline
\end{tabular}

Comparison between the treatment group and the control group before surgery showed no statistical significance (P $>0.05)$, but statistically significant difference was noted 1 month after surgery $(\mathrm{P}<0.05)$, and the difference was obvious 6 months after surgery $(\mathrm{P}<0.01)$.

\section{Comparison of 6-min walking distance}

The 6-min walking distances were statistically different between groups at 1 and 6 months after transplantation $(\mathrm{P}<0.01)$, with no difference before transplantation $(\mathrm{P}=0.059$; Table 6). 
Table 6. Comparison of 6-min walking distance between the 2 groups before and 1 month and 6 months after surgery.

\begin{tabular}{lcccc}
\hline Group & Control & Treatment & $t$ value & P value \\
\hline Preoperative & $295.07 \pm 46.87$ & $312.17 \pm 89.19$ & 3.714 & 0.059 \\
One month & $320.17 \pm 52.78$ & $422.04 \pm 50.59$ & 30.970 & $<0.01$ \\
Six months & $334.27 \pm 43.75$ & $466.36 \pm 82.86$ & 45.734 & $<0.01$ \\
\hline
\end{tabular}

Comparison of 6-min walking distance between the 2 groups before surgery showed no significant difference $(\mathrm{P}=0.059)$. Comparison of 6 -min walking distance between the control group and the treatment group showed significant difference at 1 and 6 months after surgery $(\mathrm{P}<0.01)$.

\section{Mortality and readmission}

At the follow-up 6 months after the operation, 7 patients in the control group died, and 2 patients in the treatment group died. The rate of sudden death due to various reasons in the treatment group decreased during the observation (mortality: $\chi^{2}=4.58$ ). The difference between the groups was statistically significant $(\mathrm{P}<0.05)$. The readmission rate for other reasons was low and the difference was not statistically significant (Table 7).

Table 7. Mortality and readmission rates for the treatment and control groups 6 months after surgery.

\begin{tabular}{lcccc}
\hline Group & Control $(\mathrm{N}=29)$ & Treatment $(\mathrm{N}=30)$ & $\chi^{2}$ value & P value \\
\hline Number of readmissions (rate) & $9(31.0 \%)$ & $5(16.7 \%)$ & 1.68 & $>0.05$ \\
Number of deaths (rate) & $7(24.14 \%)$ & $2(6.67 \%)$ & 4.99 & $<0.05$ \\
\hline
\end{tabular}

Six months after surgery, comparison of readmission rate between the control group and the treatment group showed no significant difference $(\mathrm{P}>0.05)$. Comparison of death rate between the treatment group and the control group showed statistically significant difference $(\mathrm{P}<0.05)$.

\section{Adverse reactions}

Among the 30 patients in the treatment group, 1 experienced chest discomfort and showed ST-T changes, but spontaneous remission was achieved 15 min after physiological saline flushing. The remainder of the 29 patients had no adverse reactions, heart palpitations, chest pain, chest tightness, dyspnea, or other symptoms. None of the 59 patients had malignant tumor 6 months after treatment.

\section{DISCUSSION}

Heart failure encompasses a series of complex clinical syndromes such as heart pump function damage caused by abnormal cardiac structure or function. Once heart failure occurs, myocardial fibrosis, degeneration, necrosis, and progressive ventricular remodeling follow. This study explores the beneficial effects of umbilical cord MSCs by intracoronary transplantation in the treatment of decompensated congestive heart failure, by observing LVEF, left ventricular contractility, exercise capacity, and cardiac function before and 1 month and 6 months after treatment. The clinical symptoms resolved, and mortality rates reduced. The possible underlying mechanism is as follows. In the myocardial microenvironment, the umbilical cord MSCs proliferate and differentiate into myocardial cells. Mitchell et al. (2003) 
found that umbilical cord stromal cells had the potential of pluripotent stem cells that was 80 times more in in vitro proliferation; moreover, these cells expressed markers of stem cells including c-kit (CD117) and telomerase. Wang et al. (2004) also found that human umbilical MSCs in the presence of 5-azacytidine (5-AZA) or under the action of myocardial cells can differentiate into myocardial cell marker N-cadherin and cardiac troponin I cells. Makino (1999) reported that the MSCs showed fibroblast-like morphology before undergoing cardiomyocyte differentiation induced by 5-AZA. Cell morphogenesis changed after 5-AZA treatment. The cells were connected with the neighboring cells, formed myotube-like structures, and began to beat.

Many researchers have stated that umbilical cord MSCs differentiated into not only myocardial cells but also vascular endothelial cells in in vitro differentiation. Lenvenberg et al. (2002) showed that human umbilical cord MSCs have in vitro endothelial differentiation potential, and the endothelial marker gene expression of these cells depends on the different induction time. It is proven that endothelial cells from the umbilical cord MSCs have functional properties of migration and formation of blood vessels. Li et al. (2010) cultured umbilical cord MSCs by in vitro isolation and differentiated them into vascular endothelial cells in the presence of vascular endothelial growth factor and fibroblast growth factor.

Clinically, we also observed alleviation of the symptoms of heart failure, such as cough, chest tightness, shortness of breath, and dyspnea $24 \mathrm{~h}$ after transplantation of umbilical cord MSCs. The role of transplanted umbilical cord MSCs induced into myocardial cells and vascular endothelial cells in the myocardial microenvironment is not well clarified. The results of the BOOST II test suggest that early stem cell paracrine proliferation is more important than stem cell differentiation into new cells. Behjati (2013) also found that MSCs in the ischemic myocardium directly differentiate as well as release cytokines by paracrine mechanisms that affect the surrounding cells, leading to improved myocardial cell metabolism and alleviation of myocardial ischemia and myocardial apoptosis. Tang et al. (2005) suggest that the paracrine effects of MSCs, mainly through the secretion of angiogenic factors and stem cell-homing factor, downregulate the pro-apoptotic protein mechanism to improve left ventricular myocardial remodeling and heart function. Ischemic heart repair requires the involvement of various kinds of cells in the body; Vrtovec et al. (2011) showed that MSCs secrete a variety of stem cell chemotactic factors, mobilize various cells, and push themselves into the ischemic area to prevent cardiac enlargement, reduce scar area, and improve heart function.

We also observed no serious adverse reaction in 30 cases of transplantation. The ST-T of 1 patient increased during surgery, but cell infarction was not found on repeated angiography. The ST-T increase may be related to the intervention surgery. No complications related to transplantation occurred. Moreover, there was no new case of cancer during the 6-month follow-up.

However, owing to the short time of observation and limited number of patients, the efficacy and safety of umbilical cord MSCs in the treatment of heart failure need to be confirmed by further randomized, double-blind, controlled, evidence-based tests.

Transplantation of umbilical cord MSCs in the treatment of heart failure is a rather novel procedure, and many issues need to be addressed. Along with the development of stem cell research, umbilical cord MSCs transplantation can be considered a novel therapeutic method for preventing progression to heart failure in the future and a new direction for the treatment of decompensated systolic heart failure. 


\section{ACKNOWLEDGMENTS}

Research supported by a key project of the Science and Technology Department of Henan Province Science and Technology (\#122101310500).

\section{REFERENCES}

Ambrosy AP, Fonarow GC, Butler J, Chioncel O, et al. (2014). The global health and economic burden of hospitalizations for heart failure: lessons learned from hhf registries. J. Am. Coll. Cardiol. 27: 291-295.

Behjati M (2013). Suggested indications of clinical practice guideline for stem cell-therapy in cardiovascular diseases: A stepwise appropriate use criteria for regeneration therapy. ARYA Atheroscler. 9: 306-310.

Chih S, Tinckam KJ and Ross HJ (2013). A survey of current practice for antibody-mediated rejection in heart transplantation. Am. J. Transplant. 13: 1069-1074.

Chugh AR, Brache GM, Loughran JH, Mewton N, et al. (2012). Administration of cardiac stem cells in patients with ischemic cardiomyopathy: the SCIPIO trial: surgical aspects and viability by magnetic resonance. Circulation 126 : 54-64.

Feldman D, Pamboukian SV, Teuteberg JJ, Birks E, et al. (2013). The 2013 International Society for Heart and Lung Transplantation Guidelines for mechanical circulatory support: executive summary. J. Heart Lung Transplant. 32: 157-187.

Giamouzis G, Kalogeropoulos A, Georgiopoulou V, Laskar S, et al. (2011). Hospitalization epidemic in patients with heart failure: risk factors, risk prediction, knowledge gaps, and future directions. J. Card. Fail. 17: 54-75.

Hou P and Li JP (2007). The biological characteristics of mesenchymal stem cells and in vitro differentiation of vascular endothelial cells. J. Clin. Rehabilit. Tissue Eng. Res. 20: 4021-4024.

Lenvenberg S, Golub JS, Amit M, Itskovitz-Eldor J, et al. (2002). Endothelial cells derived from human embryonic stem cells. Proc. Natl. Acad. Sci. U. S. A. 99: 4391-4396.

Li D, Shi C, Hong JX, Meng HX, et al. (2011). Isolation of human umbilical cord mesenchymal stem cell research tissue block method. Chin. Med. Herald 24: 19-22.

Li L, Ma HY, Ma L, Du LL, et al. (2010). Human placenta derived mesenchymal stem cells to vascular endothelial cell differentiation potential. Chin. J. Pathophysiol. 26: 2142-2148.

Makino S, Fukuda, K, Myoshi S, Konishi F, et al. (1999). Cardiomyocytes can be generated from marrow stromal cells in vitro. J. Clin. Invest. 103: 697-705.

Mitchell KE, Weiss ML, Mitehell BM, Martin P, et al. (2003). Matrix cells from Wharton's jelly form neurons and glia. Stem Cells 21: 50-60.

Perin EC, Silva GV, Zheng Y, Gahremanpour A, et al. (2012a). Randomized, double-blind pilot study of transendocardial injection of autologous aldehyde dehydrogenase-bright stem cells in patients with ischemic heart failure. Am. Heart J. 163: 415-421.

Perin EC, Willerson JT, Pepine CJ, Henry TD, et al. (2012b). Effect of transendocardial delivery of autologous bone marrow mononuclear on function capacity, left ventricular function, and perfusion in chronic heart failure: the FOCUS-CCTRN trial. JAMA 307: 1717-1726.

Sbruzzi G, Ribeiro RA, Schaan BD, Signori LU, et al. (2010). Functional electrical stimulation in the treatment of patients with chronic heart failure: a meta-analysis of randomized controlled trials. Eur. J. Cardiovasc. Prev. Rehabil. 17: 254-260.

Tang TL, Zhao Q, Qin X, Shen L, et al. (2005). Paracrine action enhances the effects of autologous mesenchymal stem cell transplantation on vascular regeneration in rat model of myocardial infarction. Ann. Thorac. Surg. 80: 229-237.

Vrtovec B, Poglajen G, Sever M, Lezaic L, et al. (2011). Effects of intracoronary stem cell transplantation in patients with dilated cardiomyopathy. J. Card. Fail. 17: 272-281.

Wang HS, Hung SC, Peng ST, Huang CC, et al. (2004). Mesenchymal Stem Cells in the Wharton's Jelly of the Hutnan Umbilical Cord. Stem Cells 22: 1330-1337.

Weiss ML, Medicetty S, Bledsoe AR, Rachakatla RS, et al. (2006). Human Umbilical Cord Matrix Stem Cells: Preliminary Characterization and Effect of Transplantation in a Rodent Model of Parkinson's Disease. Stem Cells 24: 781-792.

$\mathrm{Xu}$ Y, Li CH, Meng HX, Hao M, et al. (2009). Optimization of culture condition and biological characteristics of human umbilical cord-derived mesenchymal stem cells. J. Clin. Rehabilit. Tissue Eng. Res. 13: 6289-6294.

Genetics and Molecular Research 14 (2): 3010-3017 (2015) 